\title{
Smart Tool for Integrated Chips Selection
}

\author{
Anusha Pillay \\ VIT University \\ School of Electrical Engineering \\ Vellore, TamilNadu, India
}

\author{
Kartik Sharma \\ VIT University \\ School of Electrical Engineering \\ Vellore, TamilNadu, India
}

\author{
Monica Subashini.M \\ VIT University \\ School of Electrical Engineering \\ Vellore, TamilNadu, India
}

\begin{abstract}
Today's world is the world of speed, convenience and accuracy. With the growing demand of consumer electronics and advancing technology, developers have numerous options available for selecting ICs for a particular application, which might also make finding appropriate IC a tedious task. Hence, a smart tool is developed which is software capable of searching appropriate IC based on selected performance determining parameters, such as CMRR, Slew rate, etc. Among different ICs, op amps are used for various purpose like in amplifiers, oscillators, filters, comparators, detectors and thus are one of the most widely used chips. This paper proposes a totally new method for smart selection of operational amplifiers. It describes the design of new software that could search a suitable IC depending upon the specifications entered by the user. The software is developed using $\mathrm{C}$ programming. The coding of the tool is being done on GNU environment-Dev $\mathrm{C}++$, which can also be implemented with GUI.
\end{abstract}

\section{General Terms}

Application of Computer science in Electronics

\section{Keywords} IC.

Op-amp Parameters, Smart search, Pseudo code, Selected

\section{INTRODUCTION}

To develop a particular electronic device, developer has to think not only the design but also the grass root level requirement i.e. IC. The same may also extend to LCDs, transistors, motors etc. In this tool, we have designed new software for selection of appropriate IC as asked by the developer. The selection procedure is referred to as smart search and is coded in $\mathrm{C}$ programming language. User has to enter the specifications of IC required and our smart tool will perform its search and display the results. The search considered, in this paper, is performed on a set of operational amplifiers. Their specifications have been entered in the database. We have restricted our search to six performance determining parameters for better results. They are: slew rate, power supply, input offset voltage, input bias current, temperature range.

Thus, the selection procedure described in this paper is intended as a new tool for the developers. The same selection procedure can be not only be applied for ICs, but also for LCDs, motors, transistors and can be expanded in other fields. At the end, we have presented an example on the application of the above described tool.

\section{DESIGN PARAMETERS}

\subsection{Operational Amplifiers}

Integrated circuits are classified according to their mode of operation: digital or linear. Linear ICs had been focused as our area of interest. Moreover, only those ICs are considered which are based on op amps for serving to the application of various electronic uses.

The operational amplifier is a versatile device that can be used to amplify dc as well as input signals and was originally designed for performing mathematical operations such as addition, subtraction, multiplication and integration. There are a large number of parameters [2] associated with op amps. The very first step includes selection of appropriate parameters which the developer has to keep in mind while designing an IC since the performance of an operational amplifier depends on these characteristics [50]. The effects of characteristics on op amp applications are highlighted in Table 1

Table.1. Effects of characteristics on Op amp applications

\begin{tabular}{|c|c|c|c|c|}
\hline $\begin{array}{c}\text { Op amp } \\
\text { Characteristic } \\
\text { that may } \\
\text { affect } \\
\text { performance }\end{array}$ & $\begin{array}{c}\text { DC } \\
\text { Amplifier }\end{array}$ & $\begin{array}{c}\text { AC } \\
\text { Amplifier }\end{array}$ & $\begin{array}{c}\text { DC } \\
\text { Amplifier }\end{array}$ & $\begin{array}{c}\text { AC } \\
\text { Amplifier }\end{array}$ \\
\cline { 2 - 5 } Output & $\begin{array}{c}\text { Small } \\
\text { Output }\end{array}$ & $\begin{array}{c}\text { Large } \\
\text { Output }\end{array}$ & $\begin{array}{c}\text { Large } \\
\text { Output }\end{array}$ \\
\hline $\begin{array}{c}\text { Input bias } \\
\text { current }\end{array}$ & Yes & No & May be & No \\
\hline $\begin{array}{c}\text { Offset current } \\
\text { Input offset } \\
\text { voltage }\end{array}$ & Yes & No & May be & No \\
\hline Drift & Yes & No & May be & No \\
\hline $\begin{array}{c}\text { Frequency } \\
\text { Response }\end{array}$ & No & Yes & No & Yes \\
\hline Slew rate & No & No & Yes & Yes \\
\hline
\end{tabular}

\subsection{Op amp Characteristics}

In this work, we have used six parameters to select the best suitable IC as required by the consumers as described in reference papers [3-5]. The importance of the parameters is described below.

1. CMRR: It is a measure of the degree of matching between two input terminals. The larger the value of CMRR, the better 
is the matching between the input terminals. The CMRR value listed on the datasheets are for open loop configuration.

2. Slew rate: The maximum rates of change of voltage with respect to time, usually measured in $\mathrm{V} / \mu \mathrm{s}$. Higher slew rate op amps are advantageous.

3. Input offset voltage: It is the differential input voltage that exists between two input terminals of an op amp without any external inputs applied.

4. Input bias current: It can cause a significant output offset voltage which is desired to be as low as possible.

5. Power supply: Operational amplifiers are susceptible to changes in the power supply.

$$
\begin{aligned}
& \text { a. Single power supply } \\
& \text { b. Dual power supply }
\end{aligned}
$$

6. Temperature range: Operational amplifiers can operate over a set of temperature ranges. They are highly temperature sensitive.

\section{TOOL DESCRIPTION AND DESIGN}

The software is designed on Dev C++. Dev-C++ is a free integrated development (IDE) distributed under the General Public License for programming in $\mathrm{C}$ and $\mathrm{C}++$ [6-8]. The entire process of the smart tool is depicted in Figure.1.Flow Chart-Smart Tool.

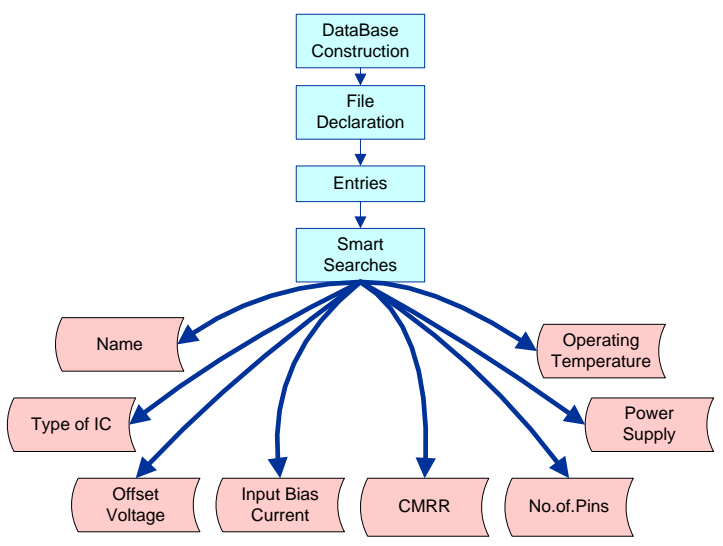

Figure 1: Flow Chart -Smart Tool

\subsection{Database construction:}

In order to perform the smart search, we had developed the database using 66 datasheets of operational amplifiers. The datasheets are given in the references [9-48]. A user-defined type was created and declared the parameters as cmrr, pin, tlow, thigh, type, psupply, slew, iov, ibc, cmrr, icname[name_max], desc (700), pwsupply (20) as its members.

\subsection{File declaration:}

- A file had been created for the storage of specifications of various ICs.

- A binary file named 'data.dat' is used to store all the data.

- Using a file pointer 'fp', data from program is stored in 'data.dat' in binary form.

- Later, the same file is used to import data in the program to perform searches. The text book referred in [1] was widely used in programming.

\subsection{Making entries:}

The entries are made using a function display after completing the process of file declaration. In the function display, an array temp(i) is created. It is an array of structures which is already defined in section A. The entries are made and it gets stored in the array.

\subsection{Smart searches:}

Thus, the database is constructed; the next essential step is to define various searches that can be performed. Here, in this tool, we have declared only eight search functions to reduce complications in searching an IC. The search screen is shown in Figure.2. Smart Search Screen.

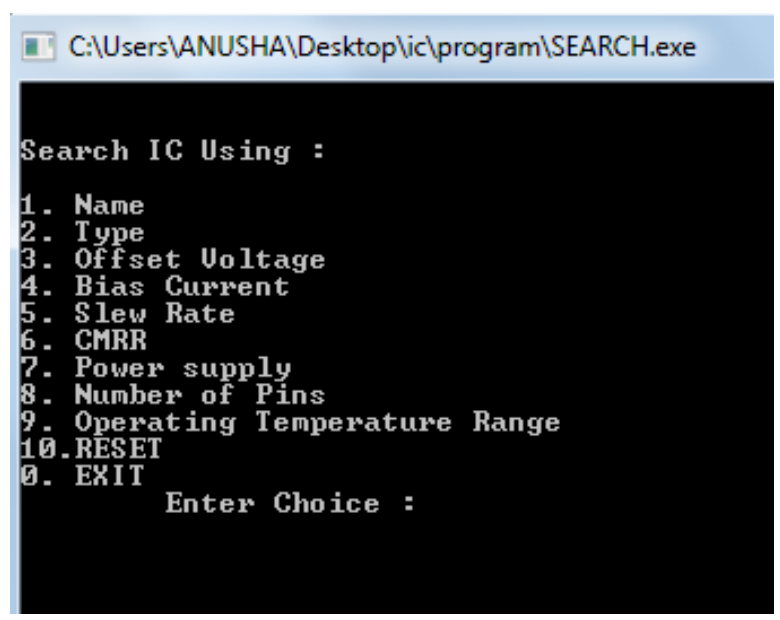

Figure 2: Smart Search Screen

Search 1: It is based on the name of IC.

Search 2: It is based on the type of IC. On the basis of our area of interest, we have used the following type of IC:

a. Differential IC

b. Classification of IC on the basis of gain of the op amp

c. High performance IC

d. Low cost IC

e. Low noise IC

f. Power op amp

Search 3: It is based on the offset voltage of the op amp.

Search 4: It is based on the input bias current of an op amp.

Search 5: It is based on CMRR.

Search 6: It is based on Power supply.

Search 7: It is based on number of pins in an IC.

Search 8: It is based on operating temperature range.

\subsection{Search results:}

After performing the smart search, the tool displays the suitable IC satisfying all the specifications and the requirements as entered by the user. It will display the name, type and all the parameters along with the description of the IC. Thus the software is designed. 


\section{RESULTS}

We have explained the entire process using a search for AD8067 which is used in automobile, telecommunication, medical appliance, industry control, computer and its peripheral, consumption electronics etc.

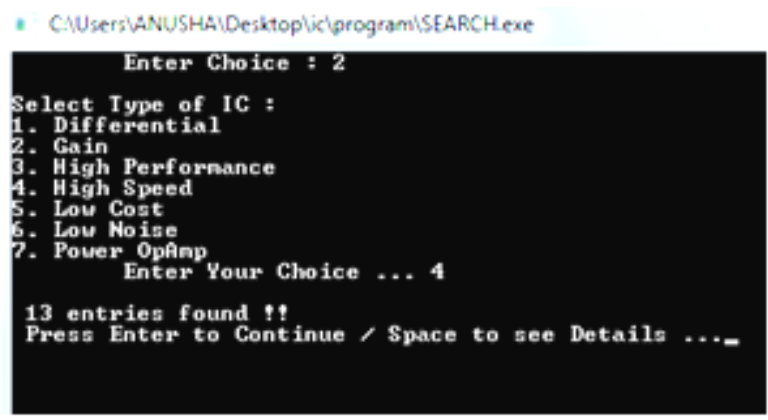

Figure 3: High Speed IC Choice entry

The search is performed based on type of the IC. Because it is used for all high speed applications we have selected type no. 4 high speed IC which is shown in Figure.3.High Speed IC Choice entry. We know that AD8067 is a unique IC with 5 pins.

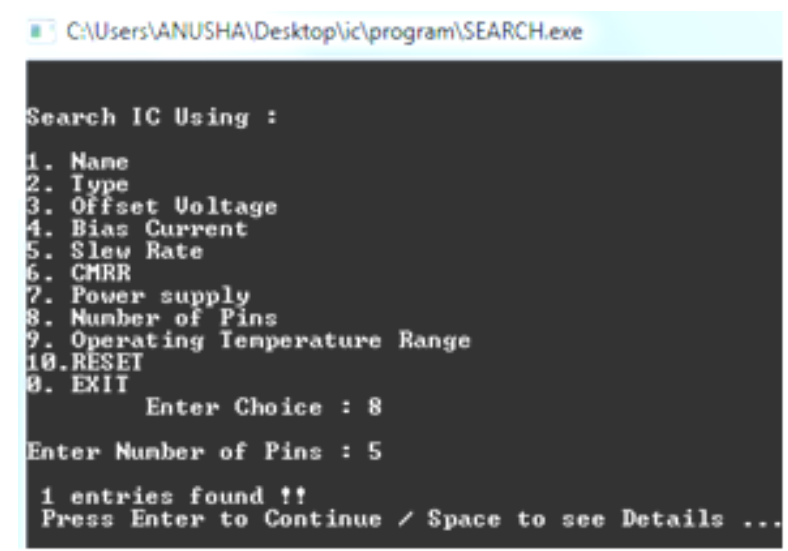

Figure 4: Smart Tool- Choice Entry Window

The next search is based on number of pins. The screenshot for the pin search is shown in Figure.4.Smart tool-Choice Entry Window. Similarly, the search can also be done based on operating temperature range, offset voltage, bias current, slew rate and CMRR.

The software now displays the required IC with all the specifications as shown in Figure.5. Smart Tool-Selected IC

\section{PERFORMANCE ANALYSIS}

The tool is a standalone application. It's a hastle free operation and hence there is no need of installation as in followed by other existing tool [49].

The entries depend on specific range as well as a specific value. The tool is user-friendly such that it enables the inclusion of new datasheets in the database.

It is a very useful tool for beginners, students, researchers and even a layman because every time an entry made by the user would narrow down the search.

The search is also done based on the number of pins (5-pin IC) and this search in unique in our tool when compared to already available tools in market [51].

The tool would select a specific IC for the specifications mentioned as well as all the available ICs within that range.

Special categories in search are available in this tool such as high speed IC, high performance IC, low cost IC, low noise and gain based

\section{CONCLUSION AND FUTURE WORK}

The discussed methodology and the designed tool is a very useful tool for beginners, students, researchers, commercial industries and laboratories for the design and development of applications.

There is no need for the users to sit for hours and go through the datasheets or surf through the internet and brainstorm their minds just to find an appropriate IC for their product or experiment. The coding has been done in GNU environment.

The future efforts would be to develop with other user friendly soft wares available. Moreover, we have used specific set of 66 ICs, we will try to broaden our area of work.

It is possible to expand this smart search to motors and appliances which finds their use in various applications. 


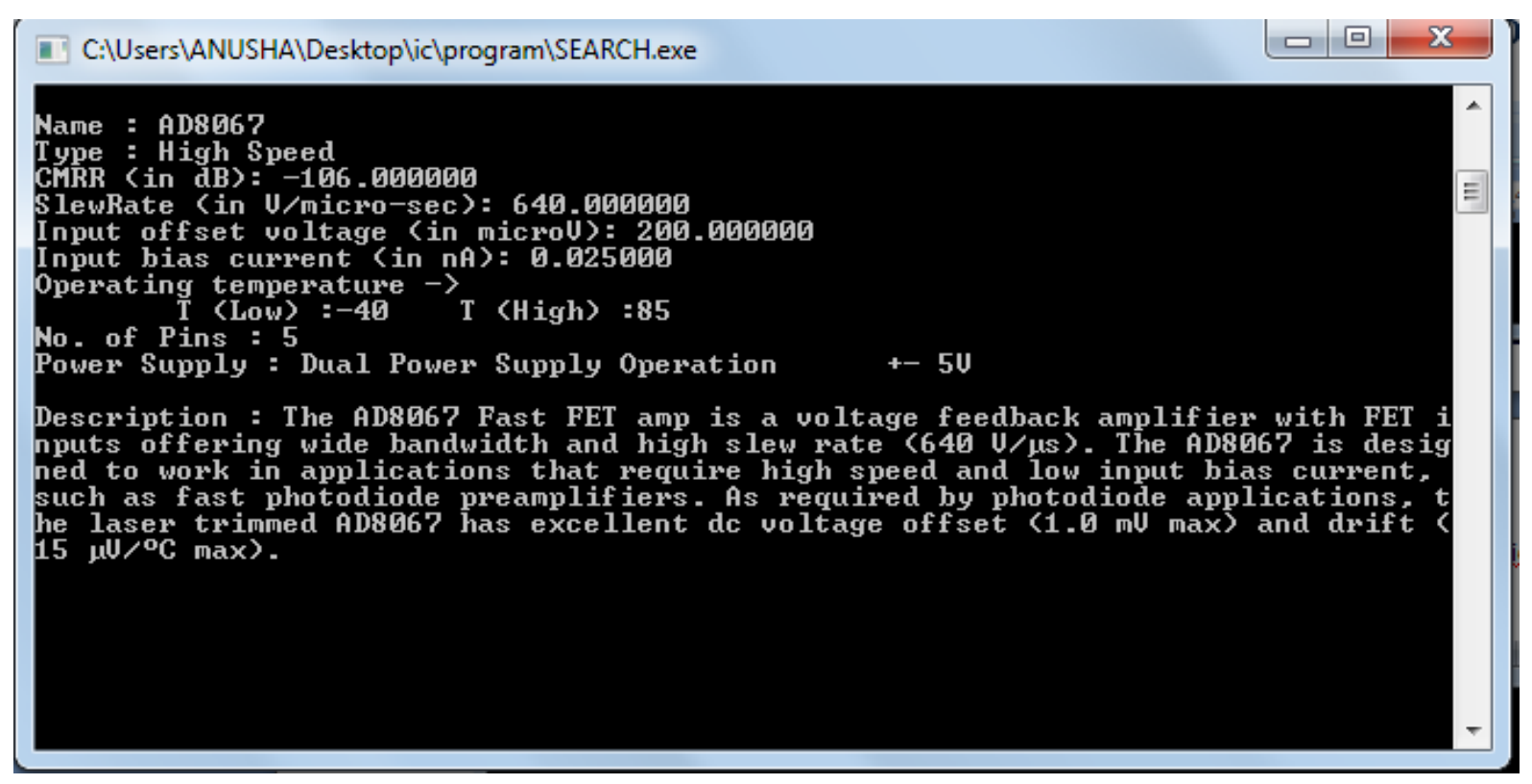

Figure 5: Smart Tool-Selected IC

\section{ACKNOWLEDGMENTS}

The work was supported by School of Electrical Engineering, VIT University, India.

\section{REFERENCES}

[1] Sumita Arora, Computer Science with C++, 9th ed, Dhanpat Rai Publications (P) Ltd, 2012.

[2] Ramakant A. Gayakwad, Op-Amps and linear integrated circuits, 4th ed, PHI learning (P) Ltd, 2010.

[3] Palumbo .G and Pennisi. S, "Performance parameters of current operational amplifiers", Proceedings of ICECS '99, IEEE International Conference , 1615 - 1618 ,vol.3 , 1999.

[4] Dinovitzer. A, "The Mooring Selection Guide (MSG) software", OCEANS '97, MTS/IEEE Conference Proceedings, oct 1997.

[5] Linear stock list IC's website Available: http://LinearIC'sOPAMPS.htm.

[6] Php

website.Available : http://php.net/manual/en/function.fseek.php.

[7] Mycpluscom website.Available : http://www.mycplus.com/tutorials/c-programmingtutorials/file-handling/

[8] Cplusplus website.Available : http://www.cplusplus.com/reference/cstdio/tmpfile/.

[9] “AD795" datasheet Lower power, lower noise, precision FET Op Amp.

[10] “AD8000”datasheet 1.5 GHz ultrahigh speed Op Amp.

[11] "AD8001" datasheet $800 \mathrm{MHz}, 50 \mathrm{mw}$ current Feedback amplifier.

[12] "AD8002" datasheet Dual $600 \mathrm{MHz}, 50 \mathrm{mw}$ current feedback amplifier.
[13] “AD8003” datasheet Triple 1.5 GHz Op Amp.

[14] "AD8004" datasheet Quad 3000 V/ms, 35 mW Current Feedback Amplifier.

[15] “AD8007 / AD8008” datasheet Ultralow Distortion High Speed Amplifiers.

[16] "AD8011" datasheet 300 MHz, 1 mA Current Feedback Amplifier.

[17] "AD8013" datasheet Single Supply, Low Power, Triple Video Amplifier.

[18] "AD8014" datasheet $400 \mathrm{MHz}$ Low Power High Performance Amplifier.

[19] "AD8017" datasheet Dual High Output Current, High Speed Amplifier.

[20] "AD8022" datasheet Dual High-Speed Low-Noise Op Amps.

[21] "AD8023" datasheet High Current Output, Triple Video Amplifier.

[22] “AD8033 / AD8034 “datasheet Low Cost, 80 MHz Fast FET TM Op Amps.

[23] "AD8047/ AD8048" datasheet $250 \mathrm{MHz}$, General Purpose Voltage Feedback Op Amps.

[24] "AD8065 / AD8067" datasheet High Performance, 145 MHz Fast FET ${ }^{\mathrm{TM}}$ Op Amps.

[25] “AD8065/AD8066*” datasheet High Performance, 145 MHz Fast FET тм Op Amps.

[26] "AD8067" datasheet High Gain Bandwidth Product Precision Fast FET TM Op Amp.

[27] “AD8072/ AD8073” datasheet Low Cost, Dual/Triple Video Amplifiers.

[28] "AD8074/AD8075" datasheet $500 \mathrm{MHz}, \mathrm{G}=+1$ and +2 Triple Video Buffers with Disable. 
[29] "AD8099" datasheet Ultralow Distortion, High Speed $0.95 \mathrm{nV} / \sqrt{\mathrm{Hz}}$ Voltage Noise Op Amp.

[30] "AD80131" datasheet Low-Cost, High-Speed Differential Driver.

[31] “ADA4896/ADA4897” datasheet $1 \mathrm{nV} / \sqrt{\mathrm{Hz}}$, Low Power, Rail-to-Rail Output Amplifiers.

[32] “AD8538" datasheet Low Power, Precision Auto-Zero Op Amp.

[33] "ADA4817-1 /ADA4817-2" datasheet Low Noise, 1 GHz Fast FET Op Amps.

[34] "ADA4310-1" datasheet Low Cost, Dual, High Current Output Line Driver with Shutdown.

[35] "ADA4856-3" datasheet Single-Supply, High Speed, Fixed $\mathrm{G}=+2$, Rail-to-Rail Output Video Amplifier.

[36] "ADA4857-1/ ADA4857-2" datasheet Ultralow Distortion, Low Power, Low Noise, High Speed Op Amp..

[37] "AD859-3" datasheet Single-Supply, Fixed G = 2, High Speed, Video Amplifier with Charge Pump.

[38] "ADA4860-1" datasheet High Speed, Low Cost, Op Amp.

[39] “ADA4861-3" datasheet High Speed, Low Cost, Triple Op Amp.
[40] "ADA4862-3" datasheet High Speed, G = +2, Low Cost, Triple Op Amp.

[41] "ADA4898-1" datasheet High Voltage, Low Noise, Low Distortion, Unity Gain Stable, High Speed Op Amp.

[42] "ADA4899-1" datasheet Unity Gain Stable, Ultralow Distortion, $1 \mathrm{nV} / \mathrm{Hz}$ Voltage Noise, High Speed Op Amp.

[43] "LF353" datasheet Wide Bandwidth Dual JFET Input Operational Amplifier.

[44] “LH0032 / LH0032C”Ultra Fast FET-Input Operational Amplifier.

[45] "LF155-LF255-LF355 /LF156-LF256-LF356/LF157LF257-LF357"datasheet wide bandwidth single j-fet operational amplifiers.

[46] "MCP6001/1R/1U/2/4" datasheet 1 MHz, Low-Power Op Amp.

[47] "OP-37" datasheet low noise, precision, High speed Operational amplifier.

[48] "LT1970" datasheet 500mA Power Op Amp with Adjustable Precision Current Limit.

[49] TI OPAMPS SELGUIDE

[50] http://services.eng.uts.edu.au

[51] http://www.microchip.com/ParamChartSearch 